\title{
Correction to: Outcome of Repeat Pulmonary Metastasectomy
}

Marcus Krüger, Katharina Franzke, Taufiek Konrad Rajab, Fabian Nadler, Moritz Möbius-Winkler, Norman Zinne, Daniel Schulz, Miriam Möller, Wolfgang Schütte, Michael Ermitsch, Bassam Redwan, Olaf Schega, and Christian Biancosino

\section{Correction to: Chapter "Outcome of Repeat Pulmonary Metastasectomy" in: Adv Exp Med Biol - Clinical and Experimental Biomedicine, https://doi.org/10.1007/} 5584_2021_635

The chapter was inadvertently published with an incorrect chapter title, which has now been corrected to read as "Outcome of Repeat Pulmonary Metastasectomy". 